\title{
AN UPDATING PRODUCTION LINE SYSTEM FOR NATIONAL TOPOGRAPHICAL DATABASE AT 1:50K OF CHINA BASED ON DIGITAL MAP GENERALIZATION
}

\author{
ZHAO Renlianga, ${ }^{\mathrm{a} *}$ CHEN Jun ${ }^{\mathrm{a}}$, WANG Donghua ${ }^{\mathrm{a}}$, SHANG Yaoling ${ }^{\mathrm{a}}$, WANG Zhongxiang ${ }^{\mathrm{a},}$ LI Xuemei ${ }^{\mathrm{a}}$,AI Tinghua ${ }^{\mathrm{b}}$ \\ ${ }^{a}$ National Geomatics Center of China, 28 Lianhuachixilu, Haidian, Beijing, 100830,China-(zhaorl@sina.com) \\ ${ }^{\mathrm{b}}$ Wuhan University, Luojia Hill,Wuhan 430072,China
}

\section{ICWG IV/VIII}

KEY WORDS: Geospatial data updating, Digital map generalization, Rule, Multi scale, Production system

\begin{abstract}
:
This paper is focused on applying digital map generalization into the updating of geo-spatial database from large scale in the collaborative updating environment for SDI, an updating production line system for national topographical database at 1:50k of China is introduced. Based on the requirements analysis and review, we analyze the key factors for implementing updating geospatial data from large scale including technical and non-technical factors, followed by the general strategy of digital map generalization in practical production environment. In fact the most important factor is recognized that it is very difficult to establish generalization rules for production systems. We emphasized on this factor in our work and established a set of rules or constrains for scale topographical database updating 1:50k scaled data from 1:10k scaled data in a full digital environment mainly based on map specifications. Finally, the production line system structure is designed and the practical implementation is given.
\end{abstract}

\section{INTRODUCTION}

In the past decades, many geospatial databases at various scales have been established at national, regional and municipal levels over the world. The most critical problem faced by national mapping agencies is the frequent updating of their databases[Chen, 2002,Heipke, 2002]. An attractive solution is to update geospatial data at smaller-scales by deriving information from frequently updated larger-scale geospatial data through generalization-based production method. In this way, ideally only one detailed database should be frequently updated and geospatial data at smaller scales are only updated on demand using that database.

Since 60s in the last century, a lot of models and algorithms for map generalization have been presented. The researches involve the generalization operation of point, line, and area features. Also, some researchers explored the operators, framework, and knowledge rules of automatic map generalization. Some of them can be employed as edit tools of data handling. Despite the progress in theory/concepts and methodology, few comprehensive are available for automatic digital map generalization although some attempts, such as ASTRA and CHANGE have been made, now some GIS vendors such as ESRI, Integraph and Laser-Scan have recently added some generalization function to their products, especially, no practical comprehensive system directly oriented to the whole geospatial data updating production line is available.

In this paper, an updating production line system for national topographical database at $1: 50 \mathrm{k}$ of China is introduced. The requirements of the application of map generalization into topographical database updating are analysed firstly in Section 2. Based on the requirements analysis and review, in Section 3, we analysed the key factors for implementing updating geospatial data from large scale including technical and nontechnical factors, followed by the general strategy of digital map generalization in practical production environment. In fact the most important factor is recognized that it is very difficult to establish generalization rules for production systems. We emphasized on this factor in our work and established a set of rules or constrains for topographical database updating at $1: 50 \mathrm{k}$ scaled data from $1: 10 \mathrm{k}$ scaled data in a full digital environment based on map specifications. Finally, the production line system is designed and the practical implementation is given in Section 4.

\section{CHALLENGE OF UPDATING FROM LARGE SCALE DATA SOURCES}

\subsection{Demands of multi scale geo-spatial database updating}

In the past twenty years, the main task of geo-spatial database is to establish digitalized geo-spatial databases. Recently, however, due to the huge requirements of geo-spatial updating, the updating issues have attracted more and more researchers and engineers, many efforts have been made in the change detection and extraction, conflict detection and resolution, automatic map generalization, feature extraction from images and so on [Cooper and Peled, 2001]. 
For instance, national multi scale geo-spatial databases ranged from 1: 4 million to 1:50000 have been established in China, and regional multi scale geo-spatial database varied from scale 1:10000 to 1:1000 have also been established in most provincial regions. However, due to the rapid economic construction and society development, geo-spatial features such as rivers, roads, resident buildings often change, so current geo-spatial databases become older and older, and become difficult to be applied in the all kinds of applications. More and more users intensively require geo-spatial data providers to keep geo-spatial database up to date.

In order to provide up to date geo-spatial data, many countries have started national plans of geo-spatial database updating. United States of America has made a large plan of national mapping plan and promised to keep the geo-spatial data update every 7-10 days. In China, the updating project of National Fundamental Geographical System 1:50000 database started in 2006. In this project, the scale 1:50000 data will be updated in the next five years, the update rang will be covered the whole country other than western of China.

As described in the previous section, there are two methods for updating geo-spatial databases. The direct method is basic for the geo-spatial database, but its cost of time and economy is very high. In fact, because of the inherent links between multi scale geo-spatial databases, the smaller scale database can be updated with the larger scale one. In other words, it is not necessary to use the direct method to update geo-spatial database at each scale. In the practical surveying and mapping, the larger scale databases often have shorter updating period than the smaller scale ones. In China, for example, geo-spatial data at scale 1:10000 are got to update every 3-5 years, but the updating period of the scale 1:50000 is 5-10 years. Therefore, it is reasonable to update the smaller scale database with the larger scale one since its cost of time and economy is less than the direct updating method.

The huge requirements have attracted lots of researchers from many fields including GIS, surveying, and spatial database. Especially, more efforts are directly from researchers in the field of map generalization since this method of updating between multi scale databases is based on map generalization.

\subsection{Technological difficulty in updating based on map generalization}

It has been recognized that updating smaller scale geo-spatial data with larger scale geo-spatial data is one of objects and tasks of map generalization in current information age [Li, 1999]. With the development of pattern recognition, computational geometry, spatial database and other information technologies, map generalization is becoming automatic and intelligent. The data result of map generalization can be used as the updated data result. Also, as another way of updating, change information can be generalized at first and then update the smaller scale data. Therefore, geo-spatial data updating require intensively map generalization technologies in the digital environment, in fact, this requirement has been one of main drives for the development of map generalization[Kilpeläinen and Sarjakoski, 1995; Badard, 1999].

Since 60s in the last century, a lot of models and algorithms for map generalization have been presented. The researches involve the generalization operation of point, line, and area features.
Also, some researchers explored the operators, framework, knowledge rules of map generalization (Li et al., 2004). Some of them can be employed as edit tools of data handling. Some commercial GIS software such as ARCINFO presented related tool package for generalization based on these researches (Lee, 2001). However, it should be recognized that these research results have not been resolved the problems of map generalization completely, as Jones pointed out, the whole field of automated generalization is still in quite an immature state (Jones, et. al. 2000).

In fact since map generalization walked into in the digital environment, the contents and ranges of map generalization has changed greatly, for example, the emphasis has not been the generalization of paper maps, but become the visualization and application analysis of geo-spatial data. In the situation of multi scale updating, map generalization technologies are also completely different from the traditional map generalization, there are some problems to be resolved, for example, it should be considered to combine the change detection and map generation operations, also handle the relation between the old and new geo-spatial data, and so on.

\section{GEO-SPATIAL DATABASE UPDATING BASED ON MAP GENERALIZATION}

\subsection{Primary rules}

Geo-spatial database updating is a complex procedure. Especially, updating based on map generalization involves lots of data sources and materials. It is reasonable to make basic rules as starting point for the desigment in order to decompose the complexity. Based on the requirements of in multi scale geo-spatial database based on map generalization and considering the technological level of map generalization, the possible rules should be considered including at least the following points.

\section{(1) Balance between spatial database and mapping}

As known, spatial database is different from paper or visual digital maps, for example, more aspects about cartography such as displacement are necessary to be considered in the mapping procedure, but not for a spatial database. Therefore, while updating a geo-spatial database, one may not consider about much aspects about the cartography effect.

(2) Combination of automatic and human-computer interactive operation

Because the whole field of automated generalization is still in quite an immature state (Jones, et.al. 2000), a practical and best choice is to make the combination of automatic operation and human-computer interactive. In practical situation, many operators of generalization could not be applied to all cases, so both automatic and interactive operations are provided.

\section{(3) Integration of different updating procedures}

Geo-spatial updating includes many procedures, for example, data sources handling, change detection and extraction, edit of generalization, confliction detection and resolution, quality check and so on. For an operator, to some degree, his working 
efficiency will be dependent on the integration of these procedures in the same system. So, a practical system must be powerful in the integration of geo-spatial database updating.

(4) Visual simulation of traditional generalization work environment

This point focuses on the links of traditional paper maps and digital work environment. In traditional way of paper maps, many generalization procedures have been used for many years, and become customs of human operators, so the new digital updating environment should have a good links to the traditional environment. For instance, in the visual environment, the larger scale geo-spatial data can be viewed as "a paper map of updating source" to be placed on the bottom of the smaller scale old data as transparent display.

\subsection{Basic principle}

Theoretically, geo-spatial updating based on map generalization is mainly dependent on two kinds of critical technologies: map generalization and change detection. Possible change information can be detected with change detection, while the new updated data can be generated with map generalization. Both of them are consisted of the core technologies for the geospatial database updating. Generally, geo-spatial database updating can be described as the following: matching the larger scale and smaller scale data and making overlay analysis to detect all possible changes; then selecting change information from larger scale based on map generalization rules , simplifying the corresponding features, detecting conflictions between features; finally checking data quality and updating the old smaller scale database. Figure. 1 shows the basic principle of geo-spatial database updating.

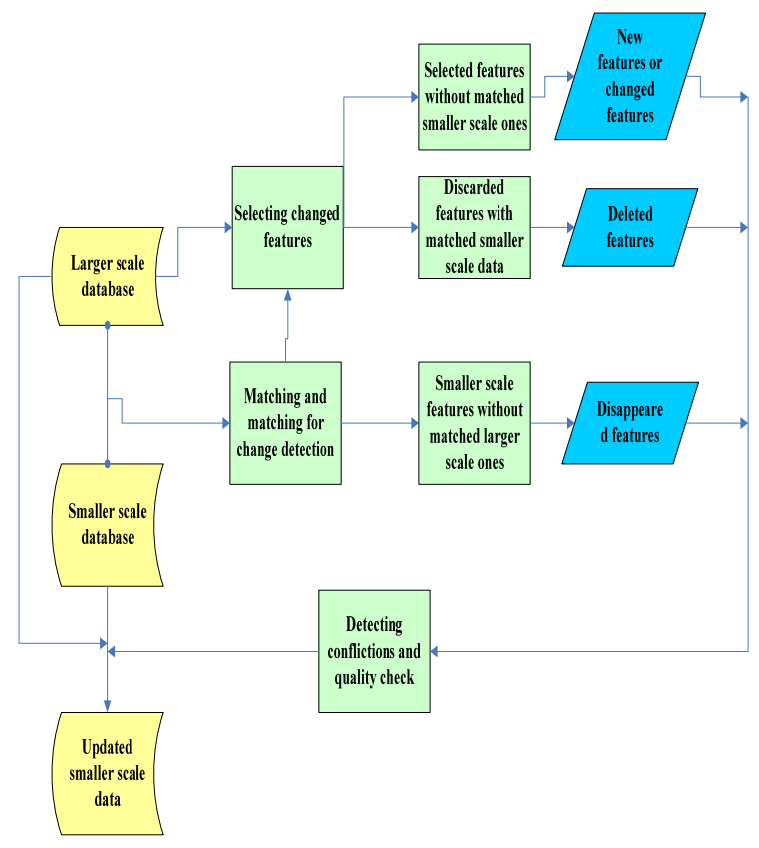

Figure 1. Basic principle

\subsection{Technical work flow}

According to the principle described above, the technical work flow of geo-spatial databases updating can be designed as fig2.
The main steps includes as follows: (1) acquire updating sources; (2) analyze the data source and determine whether they can be used; (3) pre-handle larger scale data, including format transformation, map projection transformation and edge matching and so on; (4) integrate all used data source into a visual environment and make overlay analysis; (5) detect change and extract change information with the aid of image sources; (6) apply map generalization operation edit the smaller scale data with change information derived from larger scale data; (7) detect possible conflictions between updated features and resolve them; (8) update the smaller scale data and record the changed information.

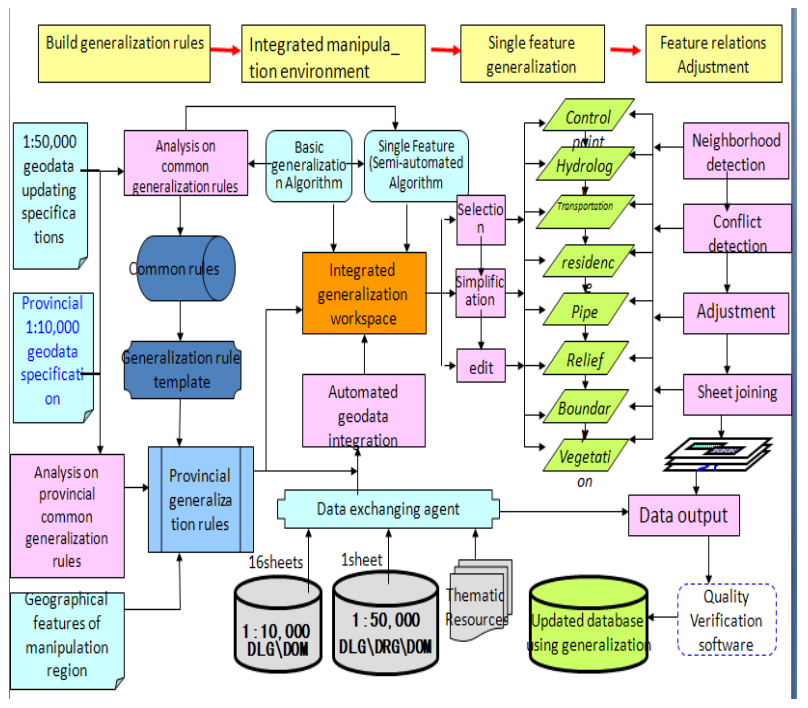

Figure 2. Technique work flow

\section{IMPLEMENTATION}

\subsection{System development strategy}

According to the design described in Section 3 and the practical requirements in a given satiation, a corresponding system can be developed to perform the task of geo-spatial database updating. Currently, some commercial softwares have developed some generation operators, but there are no mature commercial software used for geo-spatial database updating based on map generalization.

Therefore, it is a better choice to adopt an integrated strategy for developing such system, i.e., utilize a part of functions of map operators or visual environment provided by the commercial softwares, and develop the other parts based on component object technology independently.

\subsection{Implementation}

In order to meet the practical requirements of National 1:50000 geo-spatial database updating, a system is developed and implemented according to the above design and development strategy, shown in Figure 3. 




Figure 3. National 1:50000 geo-spatial database updating system based on map generalization

The system has following fundamental functions, (1) data prehandling for format transformation, map projection, data layers reorganization; (2) geometry and attribute editing; (3) change detection, feature selection and generalization; (4) detecting confliction among features and data quality check.

The production line system was applied in 24 provincial production agencies and over 500 suites are installed and put into data updating production of the project of $1: 50 \mathrm{k}$ topographical database updating. So far now, about over 3000 map sheets of 1:50k topographical data are produced using this generalization software with high production efficiency.

\section{DISCUSSION AND FUTURE WORK}

Updating is an essential step in geo-spatial database life cycle. If data are not regularly updated the results and decisions deduced from spatial analysis are unreliable. However, realizing this step is a very difficult task. The updating of geospatial databases is becoming a big challenge for many national and local surveying and mapping organisations in the world.

In this paper, the demands of updating based on generalization are analyzed in detail, and the outline of geo-spatial databases updating is described based on map generalization technology, including the basic principle, work flow and implementation. The practical application shows generalization-based updating method is very promising for multi scale database updating, especially in China for the integrating national level database resources and regional level database into the whole national spatial data Infrastructure in the future.

However, much additional work is still required. A more thorough investigation in the generalization process is required, change detection, map generalization, confliction detection and updating quality check operation require more automatic and intelligent.

\section{REFERENCES}

C.B. Jones, A.I. Abdelmoty, M.E. Lonergan, P. van der Poorten and S. Zhou 2000,"Multi-scale spatial database design for online generalization". 9th International Symposium on Spatial Data Handling, Beijing, International Geographical Union, 7b.34-44, 2000

J. Chen, Z.L. Li, J. Jiang, R.L. Zhao, 2002,“Key Issues of Continuous Updating of Geo-spatial Databases”, Geomatics World, 2(5),1-5

C. Heipke, 2002, "Requirements for modern GISs", In: Proceedings of ISPRS Commission II Symposium, CHEN Jun, JIANG Jie Eds, pp.173-179, Xi’an, Aug.20-23, 2002

A. Cooper, and A. Peled, 2001, "Incremental Updating and Versioning”. Proceedings of the 20th International Cartographic Conference, pp. 2806-2809, Beijing, August, 2001

Z. L. Li, 1999, "Scale: A fundamental dimension in spatial representation". Proceedings of the International Symposium on Digital Earth, Eds. G. Xu and Y. Chen . ISDE, Beijing, China. Science Press, Beijing, China, 1999, 1:533-538.

T. Kilpeläinen. and T. Sarjakoski, 1995, Incremental generalization for multiple representations of geographic objects, in: Lagrange J. P. and Weibel R., GIS and Generalization, Taylor \& Francis, London, 1995, 209-218.

T. Badard and C. Lemarié, 1999, "Propagating updates between geographic databases with different scales”. Chapter 10 of Innovations in GIS VII: GeoComputation, Atkinson, P. and Martin, D. (Eds.), Taylor and Francis, London, 1999.

Z. L. Li, H. Yan, T. Ai, J. Chen.,2004, “Automated building generalisation based on urban morphology and Gestalt theory”, International Journal of Geographical information Systems, 2004, 18(5): 5113-534.

D. Lee, 2001, “Generalization in the new generation of GIS”. Proceedings of the 20th International Cartographic Conference. Beijing, 2001, 2104-2109. 